\title{
Rosso Istria: un mese dopo
}

di Enrico Miletto and Flavio Febbraro

$\operatorname{Mar} 20,2019$ | Recensioni, Storia pubblica | $\underline{0 \mid}$

औstorato - 2013

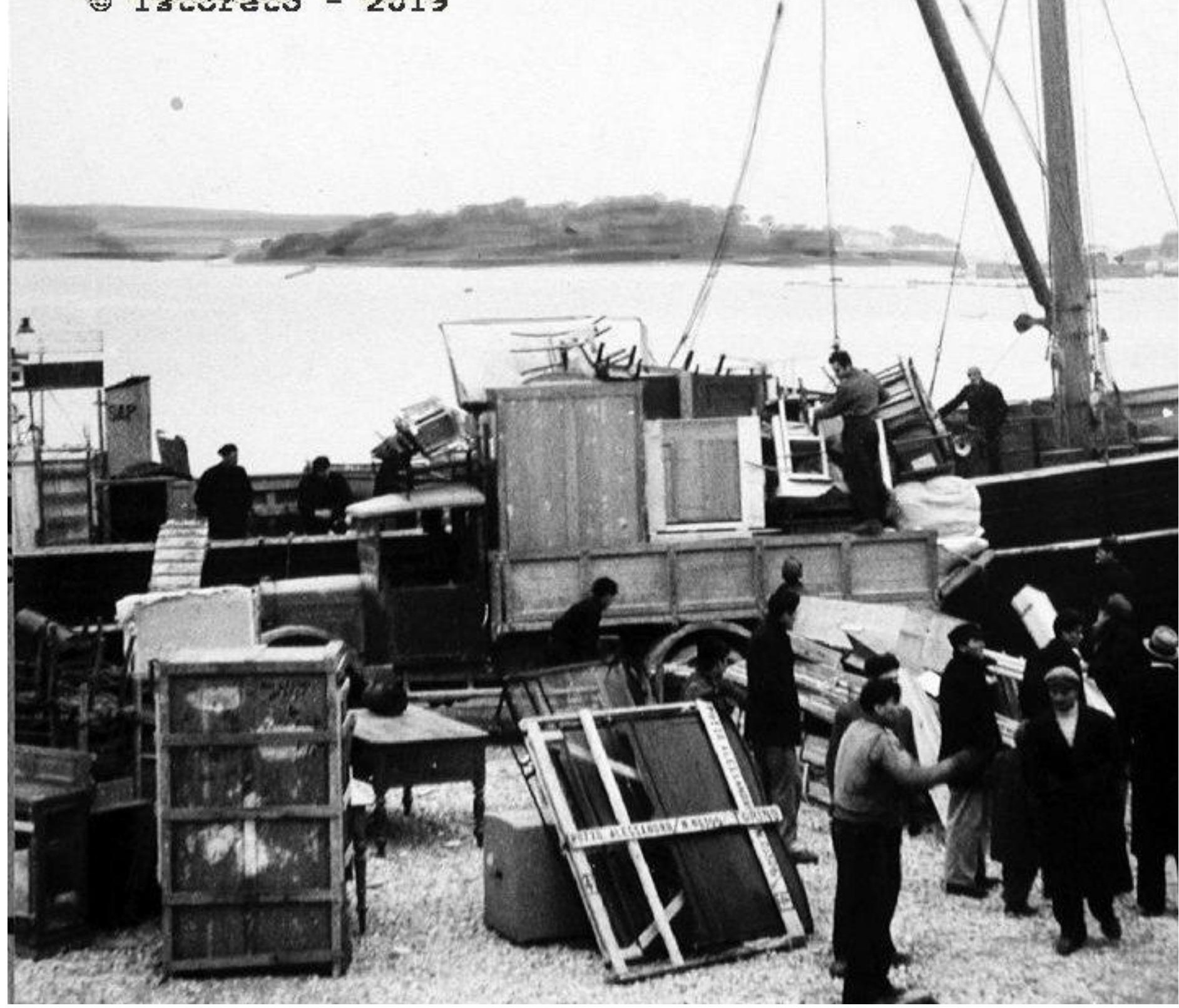

Immagine tratta da "L'esodo istriano-fiumano- dalmata in Piemonte", banca dati a cura di Istoreto, Collegamento

\section{Abstract}

A più di un mese dalla proiezione su RAI3[1] del film Rosso Istria / Red Land, abbiamo posto qualche domanda a Enrico Miletto, autore di diverse monografie e documentari sul tema dell'esodo giuliano-dalmata e coautore della banca dati L'esodo istriano-fiumano-dalmata in Piemonte. Per un archivio della memoria (Torino, Istoreto, 2014)[2] 


\section{Premessa}

La trama del film (2018, regia di Maximiliano Hernando Bruno) è così descritta nel sito ufficiale: "Siamo nel settembre del 1943, nei giorni in cui nei territori italiani martoriati dalla guerra scoppia il caos: il maresciallo Badoglio, capo del governo italiano, chiede ed ottiene l'armistizio da parte degli anglo-americani e unitamente al Re fugge da Roma, lasciando l'Italia allo sbando. L'esercito non sa più chi è il nemico e chi l'alleato. Il dramma si trasforma in tragedia per i soldati abbandonati a se stessi nei teatri di guerra ma anche e soprattutto per le popolazioni civili Istriane, Fiumane, Giuliane e Dalmate, che si trovano ad affrontare un nuovo nemico: i partigiani di Tito che avanzano in quelle terre, spinti da una furia anti-italiana.In questo drammatico contesto storico, avrà risalto la figura di Norma Cossetto, giovane studentessa istriana, laureanda all'Università di Padova, barbaramente violentata e uccisa dai partigiani titini, per la sola colpa di essere Italiana"[3].

\section{Proviamo insieme a Enrico Miletto a commentare Rosso Istria. Hai visto il film?}

$\mathrm{Si}$, ho visto il film nella proiezione cinematografica del 5 febbraio che ha coinvolto contemporaneamente circa cento sale in tutta Italia. L'evento ha rappresentato, con il successivo passaggio su RAI3, il momento più saliente della celebrazione pubblica del Giorno del Ricordo, insieme naturalmente alle cerimonie ufficiali.

\section{Al di là delle polemiche legate agli aspri conflitti di memoria che puntualmente esplodono in questi casi, che impressione hai avuto, da storico, della ricostruzione dei fatti offerta dal film?}

L'idea che ne ho avuto è quella di un prodotto che non riesce a restituire la complessità delle vicende, preferendo adottare una linea narrativa che le appiattisce. Non ho riscontrato uno sforzo sufficiente per costruire la necessaria contestualizzazione all'interno della quale inserire $\mathrm{i}$ fatti, capace di fornire allo spettatore le corrette coordinate. Il contesto storico è infatti affrontato in maniera sbrigativa e, secondo me, contiene una serie di errori che portano alla restituzione di una visione distorta. Fin dall'inizio.

\section{Puoi farmi qualche esempio?}

Fin dalle prime scene si notano una serie di incongruenze. Abbiamo una foiba e, nelle sue profondità, scorgiamo delle mani e dei respiri che, presumibilmente, sono quelli di Norma Cossetto, sulla cui vicenda non intendo entrare se non per sottolinearne - ma è naturalmente un'ovvietà - la drammaticità, dal momento che siamo di fronte alla morte di una ragazza di ventitré anni, sottoposta a una serie di violenze ed efferatezze su cui negli anni si sono susseguiti racconti non sempre aderenti a quello che le fonti consentono oggettivamente di ricostruire. Ma questo, ripeto, non mette in discussione la drammaticità della vicenda e, soprattutto, la sua sostanza. Ma torniamo al film: abbiamo dunque una foiba. Poche scene dopo, quasi immediatamente, la telecamera conduce lo spettatore al Porto Vecchio di Trieste, all'interno del Magazzino 18[4], uno dei luoghi simbolo dell'esodo, quasi a volere subito mettere in chiaro come le foibe rappresentino la chiave interpretativa dell'esodo. Si tratta di una semplificazione, anche banale, che non tiene conto dei diversi fattori che costituiscono la spinta propulsiva all'esodo. Naturalmente la paura e il ricordo delle violenze subite giocarono un ruolo fondamentale nelle dinamiche dell'esodo, ma non furono i soli elementi. L'esodo appare infatti come un percorso complesso nel quale si saldano motivazioni plurime che possono essere ricondotte a elementi di natura politica, culturale, economica e sociale e quindi non solo ed esclusivamente alle foibe come invece il film si premura di sottolineare. 
Provando ad analizzare la pellicola, un altro fattore che colpisce è la pressoché totale assenza di ogni riferimento al fascismo di confine, se si eccettua un dialogo tra due contadini croati, nel quale il figlio invita il padre a non parlare italiano. Per il resto non troviamo alcun accenno alle politiche di snazionalizzazione messe in atto dal regime, al corollario di violenze e pressioni che le accompagnano e, tanto meno, alla sanguinosa occupazione dell'esercito italiano nei territori jugoslavi[5]. Assenze che contribuiscono a non avere un quadro oggettivo del contesto storico ma che, purtroppo, non sono i soli elementi a mancare all'appello nella ricostruzione proposta dal film.

\section{Scusami Enrico, ma non credi che sia troppo pretendere da un film - che deve pur ricorrere a delle semplificazioni per esigenze di sintesi e, anche, per creare partecipazione emotiva - la restituzione di tutta la complessità della realtà storica?}

Non si tratta di pretendere da un film la completezza di un saggio storico; il fatto è che le scelte omissive o le sottolineature di alcuni aspetti a discapito di altri indirizzano la narrazione verso una tesi di fondo del film, ovvero l'esistenza di un determinato progetto mirante a una pulizia etnica nei confronti degli italiani, che invece - è cosa nota, ma forse occorre rimarcarlo per completezza - non vi fu[6].

\section{Anche in questo caso puoi indicare nel film qualche riscontro di quello che stai affermando?}

Intanto la rappresentazione dei partigiani jugoslavi: ferocia e durezza sono i canoni scelti per connotarli. Caratteristiche che sembrano trovare il loro punto di saldatura in Mate, il capo partigiano protagonista della pellicola (fatto calare quasi improvvisamente da Dubrovnik e dunque da una realtà molto distante dai luoghi in cui gli eventi si svolgono). Si tratta di una rappresentazione fortemente stereotipata tesa a far emergere un carattere rozzo, violento e brutale, quasi selvaggio, al quale si accompagna un utilizzo sistematico della violenza contro la popolazione civile. Non solo italiana, ma anche croata, come accade ad esempio in una scena nella quale Mate e i suoi uomini entrano nella casa di una contadina croata accanendosi contro di lei (e la figlia che verrà violentata) poiché colpevole - a loro dire - di avere sposato un italiano. Questa caricatura quasi animalesca dei partigiani jugoslavi è al servizio di quello che appare essere il loro unico obiettivo: colpire nella maggiore misura possibile la popolazione italiana. Una tesi rafforzata anche attraverso i dialoghi di Mate e dei suoi compagni, il cui linguaggio ha un continuo, ossessivo, rimando alla volontà di eliminare gli italiani, naturalmente sinonimo di fascisti. E non va meglio ai partigiani italiani, anche loro ridotti a soggetti pressoché privi di autonomia e potere decisionale, di fronte alla suprema volontà di Mate e dei suoi. Uno schema alquanto riduttivo del rapporto che regolerà le relazioni tra partigiani italiani e jugoslavi e del quale il film non tiene minimamente conto. In tal senso mi sembra esemplificativa una battuta rivolta dallo stesso Mate a Giorgio, un giovane (segretamente innamorato di Norma Cossetto) che, rientrato dopo 1'8 settembre, decide di unirsi al gruppo di antifascisti italiani collaboratori dei partigiani jugoslavi: "Tu sei parte della mia servitù, quindi fai solo quello che dico io, hai capito italiano?".

Inoltre, quasi a contrappunto di questa rappresentazione, ci sono i tedeschi: questi ultimi appaiono svuotati da ogni tratto di brutalità e durezza e il loro arrivo è atteso quasi con ansia da alcuni protagonisti (tra cui la famiglia Cossetto), che vedono in loro l'unica possibilità in grado di contrastare le forze jugoslave, ripristinando così il mondo precedente. Relativamente all'arrivo dei tedeschi, un'altra carenza del film è senza dubbio quella di non dedicare nessun approfondimento, eccezion fatta per dei timidi e sporadici riferimenti, all'Operazione nubifragio (Wolkenbrucht) che, scattata il 2 ottobre 1943, vide due divisioni delle SS tedesche procedere alla riconquista dell'Istria, trascinando con sé violenze contro i civili, fucilazioni, incendi di villaggi e saccheggi, che non trovano alcun spazio nella pellicola. 


\section{Quindi, nel film i tedeschi sono i "liberatori"?}

Sì, è proprio così: i tedeschi sembrano fare un ingresso alla "arrivano i nostri", connotandosi quasi come una presenza salvifica. Intendiamoci: è vero che in alcune comunità contadine dell'Istria come ha messo in luce nei suoi studi Gloria Nemec[7] - si diffonde una modalità rappresentativa del partigiano jugoslavo simile a quella proposta dal film, ma si tratta di una visione che affonda le sue radici nella concezione stessa della guerra partigiana, intesa come anticipatrice di un mondo alla rovescia che avrebbe avuto la sua piena attuazione alla fine del conflitto: una sorta di stravolgimento dell'ordine costituito fino a quel momento, tale da fare accettare l'arrivo dei soldati tedeschi come quello dei salvatori. La questione è, però, che questa dimensione specifica di un determinato momento storico e di determinati luoghi è raccontata come se fosse la condizione generale, quasi a voler uniformare il contesto, connotando di un aurea quasi salvifica l'arrivo delle truppe naziste. Insomma, quello che manca in questa visione è la complessità che deve fare da sfondo alla rappresentazione delle forze in gioco, mutuata da un diverso livello della paura, elemento fondamentale su cui basare ogni considerazione.

Voglio poi aggiungere ancora un'osservazione: nei cartelli finali del film che precedono i titoli di coda sono presentate le cifre relative alle vittime delle foibe e all'esodo. Scopriamo così che nel 1943 nelle foibe morirono 1.000 persone e che nella primavera del 1945 (quando si assistette alla seconda ondata di infoibamento) le vittime furono 7.000. Riguardo ai numeri dell'esodo, viene quantificato in 350.000 persone appartenenti soltanto alla popolazione italiana, quindi senza considerare un elemento non trascurabile nell'analisi del fenomeno (che, è bene ricordare, si inserisce a pieno negli spostamenti forzati di popolazione che coinvolsero l'Europa nell'immediato dopoguerra e che videro milioni di profughi percorrere le strade del continente[8]), e cioè che insieme agli italiani lasciò l'Istria anche una quota di sloveni e croati che non erano disposti a vivere sotto il regime jugoslavo.

\section{Questo aspetto delle cifre è sempre stato oggetto di aspre polemiche: possiamo provare a fare un po' di chiarezza?}

I dati cui faccio riferimento sono quelli che si trovano all'interno del Vademecum per il Giorno del Ricordo[9] realizzato dall'Istituto regionale per la storia del movimento di liberazione nel Friuli Venezia Giulia che costituisce un ottimo strumento per chiunque voglia approfondire le tematiche del confine orientale. In sintesi, si parla di una forbice che oscilla tra le 500 e le 700 vittime per quanto riguarda il 1943 e per le 3-4.000 (tra infoibati e scomparsi) per quanto concerne la primavera del 1945. Sull'esodo giuliano-dalmata, le stime più puntuali parlano di circa 300.000 partenze al cui interno la componente italiana rappresenta, con 250.000 persone, quella più numerosa. Tengo comunque a precisare che questa "tristissima contabilità" non ha nessuna intenzione di sminuire il fenomeno e la sua gravità, con il suo corollario di dolori e sofferenze; semplicemente ci aiuta a definirne i contorni e le dimensioni.

\section{A conclusione di queste tue considerazioni, secondo te il film ha tenuto conto dei risultati della ricerca storica?}

Gli esempi che ho indicato evidenziano, secondo me, la disinformazione degli autori, che non hanno ritenuto di confrontarsi con le acquisizioni della ricerca: quest'ultima, nonostante alcune divergenze, ha comunque raggiunto un'intesa di massima se non sull'interpretazione degli eventi, quanto meno sulle cifre legate a queste complesse vicende. Ciò vuol dire che anche in questo caso, come già avvenuto per Il cuore nel pozzo[10] (la fiction Rai del 2005) e, a mio avviso, anche per Magazzino 18[11] di Simone Cristicchi, gli storici sono se non messi ai margini, relegati in una posizione di secondo piano. Un'assenza significativa - e a dire il vero piuttosto grave - che non 
favorisce la comprensione dei fenomeni di cui parliamo ma che, al contrario, contribuisce a rafforzare un drammatico vuoto di conoscenza storica. Una circostanza che, oltretutto, assume un valore particolare se si considera che il film è stato utilizzato in molte scuole italiane in occasione del Giorno del Ricordo. E quest'ultimo aspetto, legato alla scuola, è secondo me quello più critico, dal momento che catapulta di fronte agli studenti (che nella maggioranza dei casi si presume non conoscano a fondo la questione) una realtà completamente decontestualizzata che si nutre di un linguaggio proprio dell'uso pubblico della storia, con fini di persuasione dell'opinione pubblica e, in particolare, degli studenti su un'interpretazione dei fatti molto orientata politicamente.

A questo proposito, la politica è intervenuta: il 23 gennaio 2019 è stata approvata in modo bipartisan una Risoluzione da parte della VII Commissione parlamentare della Camera dei Deputati in cui si dichiara, tra l'altro, che in occasione della 75sima Mostra nazionale del cinema di Venezia è stato presentato il film Red Land-Rosso Istria che "[...] ricostruisce in modo storicamente oggettivo ed equilibrato le tragiche vicende delle foibe $e$ in particolare il martirio di Norma Cossetto, medaglia d'oro al merito civile della Presidenza della Repubblica[12]". Cosa ti senti di dire in proposito?

Non sono d'accordo che il film ricostruisca in modo "storicamente oggettivo ed equilibrato" queste vicende, proprio perché della complessità il film non dà traccia ma, al contrario, tende a semplificare e banalizzare. Detto questo, approvare una risoluzione di questo tipo vuol dire non tenere conto dei risultati della ricerca che, se si entra nei dettagli della discussione che porta all'approvazione della risoluzione[13], sembrano essere sconosciuti ai firmatari del documento.

Un altro elemento di riflessione emerso dalla lettura della risoluzione è dato dalla sua volontà di stilare una sorta di gerarchia di chi sia titolato o meno a poter parlare nelle scuole della vicenda del confine orientale. Si parla dei testimoni, delle associazioni degli esuli e, infine, degli storici - si legge nel documento - "[...] che collaborano con le universitâ" [14]. Alla luce di ciò mi sembra di poter affermare che i firmatari non abbiano tenuto conto del ragionamento storico e storiografico che in questi anni si è condotto sulle fonti, privilegiando invece la testimonianza come unico strumento di narrazione. Una visione piuttosto riduttiva, così come lo è altrettanto quella di lasciare il campo esclusivamente a coloro che sono legati alle Università. Una formula molto e troppo generale che ad esempio - almeno sulla carta - taglierebbe fuori gli Istituti della Resistenza che da anni hanno avviato una lunga stagione di ricerca che ha raggiunto risultati notevoli sia sul piano scientifico che su quello divulgativo.

Per finire, puoi darci qualche suggerimento bibliografico e sitografico per approfondire il tema?

Per chi volesse approfondire la questione del confine orientale e dell'esodo, rimando a una bibliografia sintetica (comprendente anche dei testi di letteratura) e a una sitografia che troverete di seguito.

Bibliografia sintetica

\section{Generale}

- M. Cuzzi, G. Rumici, R. Spazzali, Istria, Quarnero, Dalmazia: storia di una regione contesa dal 1796 alla fine del $20^{\circ}$ secolo, Istituto regionale per la cultura istriano-fiumanodalmata, Libreria editrice goriziana, Gorizia 2009 
- $\quad$ E. Ivetic, Istria nel tempo: manuale di storia regionale dell'Istria con riferimenti alla città di Fiume, Unione italiana Fiume; Università popolare Trieste, Centro di ricerche storiche Rovigno 2006

\section{Fascismo di confine}

- J. Pirjevec, M. Kacin Wohinz, Storia degli sloveni in Italia 1866-1998, Marsilio, Venezia 1998

- T. Sala, Il fascismo italiano e gli Slavi del sud, Irsml FVG, Trieste 2008

- M. Verginella, Il confine degli altri : la questione giuliana e la memoria slovena, Donzelli, Roma 2008

- Anna Maria Vinci, Sentinelle della patria. Il fascismo al confine orientale 1918-1941, Laterza, Roma-Bari 2011

Foibe

- E. Apih, Le foibe giuliane, (a cura di), R. Spazzali, M. Cattaruzza, O. Moscarda Oblak, LEG, Gorizia 2010

- G. Valdevit ( a cura di), Foibe, il peso del passato, Venezia Giulia 1943-1945, Marsilio, Venezia 1997

- R. Pupo, R. Spazzali, Foibe, Bruno Mondadori, Milano 2003

- R. Pupo, Trieste '45, Laterza, Roma-Bari 2010

\section{Esodo giuliano-dalmata}

- Alessandra Algostino et al., Dall'impero austro-ungarico alle foibe. Conflitti nell'area altoadriatica, Bollati Boringhieri, Torino 2009

- Marina Cattaruzza, L'Italia e il confine orientale, il Mulino, Bologna 2007

- Cristiana Colummi, Liliana Ferrari, Gianna Nassisi, Germano Trani, Storia di un esodo. Istria 1945-1956, Irsml, Trieste 1980

- Guido Crainz, Il dolore e l'esilio. L'Istria e le memorie divise d'Europa, Donzelli, Roma 2005

- Riccardo Marchis (a cura di), Una narrazione a lungo mancata. Della diaspora giulinodalmata e degli altri esodi del Novecento alla luce del tempo presente, Seb 27, Torino 2019

- Enrico Miletto (a cura di), Senza più tornare. L'esoso istriano, fiumano, dalmata e gli esodi nell'Europa del Novecento, Seb 27, Torino 2012

- Raoul Pupo, Fiume città di passione, Laterza, Roma-Bari 2017

- Raoul Pupo, Il lungo esodo. Istria: le persecuzioni, le foibe, l'esilio, Rizzoli, Milano 2005

\section{Spostamenti forzati di popolazione nell'Europa post-bellica}

- Patrizia Audenino, La casa perduta: la memoria dei profughi nell'Europa del Novecento, Carocci, Roma 2015

- Guido Crainz, Raoul Pupo, Silvia Salvatici (a cura di), Naufraghi della pace. Il 1945, $i$ profughi e le memorie divise d'Europa, Donzelli, Roma 2008

- Antonio Ferrara, Nicolò Pianciola, L'età delle migrazioni forzate: esodi e deportazioni in Europa 1853-1953, il Mulino, Bologna 2012

- Tony Judt, Postwar. La nostra storia 1945-2005, Laterza, Roma-Bari, 2017

- Mark Mazower, Le ombre dell'Europa. Democrazie e totalitarismi nel XX secolo, Garzanti, Milano 2000 
- Keith Lowe, Il continente selvaggio. L'Europa alla fine della seconda Guerra mondiale, Laterza, Roma-Bari 2013

- Silvia Salvatici, Senza casa e senza paese. Profughi europei nel secondo dopoguerra, il Mulino, Bologna 2008

\section{Letteratura esodo/esodi}

- Enzo Bettiza, Esilio, Mondadori, Milano 1998

- Heinrich Böll, L'angelo tacque, Einaudi, Torino 1992

- Alessandra Fusco, Tornerà l'imperatore. Storia di una donna istriana tra guerra ed esodo, Affinità Elettive, Ancona 2002

- Marisa Madieri, Verde acqua e La radura, Einaudi, Torino 1998

- Claudio Magris, Alla cieca, Garzanti, Milano 2005

- Claudio Magris, Microcosmi, Garzanti, Milano 1997

- Biagio Marin, La pace lontana. Diari 1941-1950, LEG, Gorizia 2005

- Guido Miglia, Dentro l'Istria. Diario 1945-1947, Tipografia Moderna, Trieste 1973

- Nelida Milani, Anna Maria Mori, Bora, Frassinelli, Milano 1998 (nuova edizione, Id., Bora. Il vento dell'esilio, Marsilio, Venezia 2018)

- Nelida Milani, Lo spiraglio, Besa Editrice, Nardò 2017

- Nelida Milani, Di passaggio, in Nelida Milani, Racconti di guerra, Il ramo d'oro editore/Edit, Trieste-Fiume 2008

- Nelida Milani, Una valigia di cartone, Sellerio, Palermo 1991

- Czesłav Miłosz, La mia Europa, Adelphi, Milano 1985

- Helga Schneider, L'usignolo dei Linke, Adelphi, Milano 2004

- Fulvio Tomizza, La miglior vita, Mondadori, Milano, 1996

- Fulvio Tomizza, Materada, Mondadori, Milano 1960

Sitografia

- G. Nemec, R. Pupo, A. Vinci, Vademecum per il giorno del ricordo, Irsml, Trieste 2019

- F. Cecotti, B. Pizzamei, Il confine orientale italiano 1797-2007, Irsml, Trieste 2014

- Insmli, La frontiera orientale negli ultimi due secoli, Insmli

- Isgrec, Dossier sul confine orientale, Isgrec, Grosseto 2014

- Enrico Miletto, Carlo Pischedda, L'esodo istriano, fiumano e dalmata in Piemonte. Per un archivio della memoria, Istoreto, Torino 2013

Note:

[1] Il film è stato proiettato in prima serata l' 8 febbraio 2019. È stato seguito da circa 871.000 spettatori per uno share del 3,7\%. I dati sono consultabili al link https://www.tpi.it/2019/02/09/ascolti-tv-venerdi-8-febbraio-ieri/

[2] Sul tema Enrico Miletto ha pubblicato: Con il mare negli occhi. Storia e memorie dell'esodo istriano a Torino (Milano, Franco Angeli, 2005), Istria allo specchio. Storia e voci di una terra di confine (Milano, Franco Angeli, 2007), Arrivare da lontano. L'esodo istriano, fiumano e dalmata nel Biellese, nel Vercellese e in Valsesia (Varallo, Isrsc Bi-Vc, 2010). È curatore del volume Senza più tornare. L'esodo istriano, fiumano e dalmata e gli esodi nel Novecento europeo (Torino, Seb 
27, 2012) e coautore dell'applicazione multimediale L'esodo istriano-fiumano-dalmata in Piemonte. Per un archivio della memoria http://intranet.istoreto.it/esodo/ (Torino, Istoreto, 2014) e dei documentari Il sorriso della Patria. L'esodo giuliano-dalmata nei cinegiornali del tempo (Torino, Istoreto-Consiglio regionale del Piemonte, 2014) e La città vuota. Pola 1947. Il suo esodo e la sua storia (Torino, Istoreto, 2017).

[3] La trama del film è consultabile al link http://www.rossoistria.it/Rosso_Istria.html

[4] Il Magazzino 18 del Porto vecchio di Trieste è un luogo simbolico e allo stesso tempo evocativo dell'esodo. Qui si trovano infatti sedie, mobili, bauli, tavoli e molti altri oggetti di vita quotidiana, lasciati dagli esuli in attesa di poterli ritirare. Ancora oggi vi sono circa 2.000 metri cubi di masserizie di ogni tipo che mai hanno potuto ricongiungersi con i loro proprietari.

[5] Sull'occupazione italiana in Jugoslavia, cfr. Eric Gobetti, Gli alleati del nemico. L'occupazione italiana in Jugoslavia (1941-1943), Laterza, Roma-Bari 2013.

[6] Su questo tema si vedano i numerosi studi di Raoul Pupo, tra i quali citiamo Raoul Pupo, Il lungo esodo. Istria: le persecuzioni, le foibe, l'esilio, Rizzoli, Milano 2005; Id. Trieste '45, Laterza, Roma-Bari 2010.

[7] G. Nemec, Un paese perfetto. Storia e memoria di una comunità in esilio: Crisignana d'Istria (1930-1960), Leg, Gorizia 1998; Ead, Un altro essere che non è un animale, vive nei boschi. Percezione del partigianato e memoria collettiva in una comunità dell'Istria interna, in D. Gagliani, E. Guerra, L. Mariani, F. Tarozzi (a cura di), Donne, guerra, politica. Esperienze e memorie della Resistenza, Clueb, Bologna 2000; Ead. Fuori dalle mura. Cittadinanza italiana e mondo rurale slavo, in M. Cattaruzza (a cura di), Nazionalismi di frontiera. Identità contrapposte sull'Adriatico nord-orientale, Rubbettino, Soveria Mannelli 2003

[8] Le espulsioni forzate riguardarono circa 13-14 milioni di persone, in particolar modo le comunità tedesche dell'Europa orientale. Il maggior numero di espulsioni coinvolse la Polonia (circa 8 milioni) e la Cecoslovacchia (circa 3 milioni), seguite da Ungheria, Romania e, in parte, Jugoslavia. Le cifre proposte si trovano in A. Ferrara, N. Pianciola, L'età delle migrazioni forzate: esodi e deportazioni in Europa 1853-1953, il Mulino, Bologna 2012, pp. 335-336, 354-356; K. Lowe, Il continente selvaggio. L'Europa alla fine della seconda Guerra mondiale, Laterza, RomaBari 2013, p. 264. P. Audenino, La casa perduta, La memoria dei profughi nell'Europa del Novecento, Carocci, Roma 2015.

[9]Il Vademecum per il Giorno del Ricordo è consultabile all'indirizzo: https://www.irsml.eu/eventi/630-vademecum-per-il-giorno-del-ricordo . I testi sono di Gloria Nemec, Raoul Pupo e Anna Vinci, le mappe di Franco Cecotti.

[10] Alberto Negrin, Il cuore nel pozzo, Rai Fiction, Rizzoli Audiovisivi, Italia, 2005.

[11] Magazzino 18 è uno spettacolo teatrale di Simone Cristicchi portato in scena nel 2013. Dal testo è stato tratto S. Cristicchi (con J. Bernas), Magazzino 18. Storie di italiani ed esuli d'Istria, Fiume e Dalmazia, Mondadori, Milano 2014.

[12] La Risoluzione è consultabile all'indirizzo

http://www.camera.it/leg18/824?tipo=A\&anno=2019\&mese=01\&giorno=23\&view=filtered_scheda $\underline{\text { commissione }=07}$ 
[13] La discussione relativa alla Risoluzione è consultabile all'indirizzo

http://documenti.camera.it/leg18/resoconti/commissioni/bollettini/html/2019/01/23/07/comunic.htm pp. 97-99.

[14] Nello specifico, la Risoluzione recita "[...] pare necessario che [...] sia più opportuno che a essere chiamati a ricordare e a spiegare nelle scuole questi tragici eventi siano i testimoni diretti di quei fatti o gli appartenenti ad associazioni di esuli istriano-giuliano-dalmati.” E ancora: “[...] garantire che, nel rispetto dell'autonomia scolastica, siano i testimoni di quelle vicende, gli appartenenti ad associazioni di esuli istriano-giuliano-dalmati insieme agli storici che collaborano con le università, ad incontrare gli studenti, al fine di trasmettere e conservare la memoria della storia e della tragedia dei confini orientali [...]". 